\title{
Antidepressant-Like Effects of Barley (Hordeum vulgare) in a Mouse Model of Reserpine-Induced Depression
}

\author{
Hamad Rashdan Ali Al-Harbi 1',2, Sameer Al Harthi², Mansour Suliman², Omar Saadah³, Ahmed Esmat², \\ Lateef M. Khan², Shahid Karim² \\ 'Medical Education and Research Center, Al-Soqour General Hospital, Oklat, Departments of 2Pharmacology and 3 Pediatrics, Faculty of Medicine, King Abdulaziz \\ University, Jeddah, Saudi Arabia, “'Department of Pharmacology and Toxicology, Faculty of Pharmacy, Ain Shams University, Cairo, Egypt
}

\begin{abstract}
Background: Depression is a mental disorder characterized by low mood and loss of pleasure or interest in usual activities and often results in cognitive dysfunction such as impairment of learning and memory. For most of the synthetic antidepressants, severe defects such as narrow spectrum, adverse reactions, high drug price, and easy recurrence exist. Barley is one of the richest sources of antioxidants; therefore, we examined whether barley has an effect on depression, learning, and memory in a mouse model of reserpine-induced depression. Methods: Mice were individually acclimated for 1 week and then treated with barley $(200 \mathrm{mg} / \mathrm{kg}, \quad$ p. o.) or/and fluoxetine $(20 \mathrm{mg} / \mathrm{kg}$, i. p.) for 4 weeks prior to reserpine treatment. Mice were then injected with a single dose of reserpine $(2 \mathrm{mg} / \mathrm{kg}$, i. p.) or vehicle $(20 \mathrm{mg} / \mathrm{kg}$, i. p.) and assessed for mouse behaviors $1 \mathrm{~h}$ prior to tests. Mouse behavior was examined in the forced swimming test, tail suspension test, hole-board test, novel place/object recognition, social interaction test, spontaneous locomotor movement (SLMA), and stereotype movement following completion of the treatment protocol. Results: There was a significant antidepressant-like effect in the forced swimming test among the barley group than in the reserpine-treated group, and these decreases were significantly attenuated to a similar extent by treatment with fluoxetine. The effect of barley on the mean duration of immobility time was significantly attenuated in comparison with the reserpine group $(P<0.05)$. Moreover, the number of head pokes was significantly increased in the barley group in comparison with the reserpine or fluoxetine group. The mean duration of immobility time in the tail suspension test was significantly reduced in mice in the barley group in comparison with the reserpine group $(P<0.05)$. Furthermore, the social behavior test indicated that mice treated with fluoxetine have a significant increase in the distance covered by the mice toward familiar ones compared to the barley group, whereas the distance measured to the stranger mice was significantly increased among those who received fluoxetine with barley in comparison with the barley group alone. Finally, novel object recognition test, spontaneous locomotor movements (SLMA), and the stereotype movements showed that barley significantly decreased time spent on exploring the novel objects as well as in stereotype movements in comparison with the reserpine group. Conclusion: We conclude that barley can ameliorate depressive-like effects. Taken together, these findings demonstrate that barley may be effective in treating patients with depression.
\end{abstract}

Key words: Antidepressant-like effects, barley, forced swimming test, novel object recognition test, social behavior test, spontaneous locomotor and stereotype movements, tail suspension test

\section{SUMMARY}

- Barley (Hordeum vulgare) green leaves were used as a natural medicine to treat depression

- Resepine causes monoamine depletion in mice thereby exhibiting anxiety and depression-like behavior

- Our findings indicate that barley decoction may demonstrate an antidepressant-like effect.

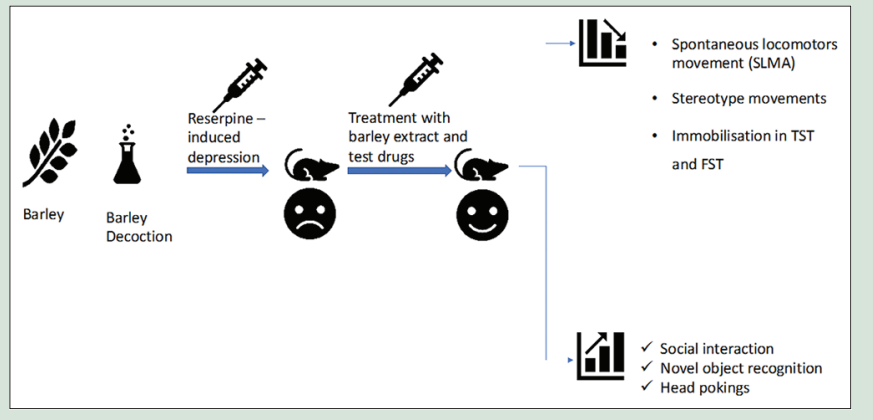

Abbreviations Used: MDD: Major depressive disorder; R: Reserpine; RFB: Reserpine, fluoxetine, and barley; B: Barley; C: Control; I: Imipramine; F: Fluoxetine; FST: Forced swimming test; TST: Tail suspension test; SLMA: Spontaneous locomotor movements;TCAs:Tricyclic antidepressants; SSRIs: Selective serotonin reuptake inhibitors; MAOIs: Monoamine oxidase inhibitors; NARIs: Noradrenergic reuptake inhibitors; SNRTs: Serotoninnorepinephrine reuptake Inhibitor; GABA: Gamma-aminobutyric acid; HPA: Hypothalamic-pituitary-adrenal; NMDAR: $\mathrm{N}$-methyl-D-aspartate receptor; VMAT: Vesicular monoamine transporter.

\section{Correspondence:}

Dr. Shahid Karim,

Department of Pharmacology, Faculty of Medicine, King Abdulaziz University, Jeddah - 21589, Saudi Arabia.

E-mail: shahid.karim@yahoo.co.in DOI: 10.4103/pr.pr_105_19

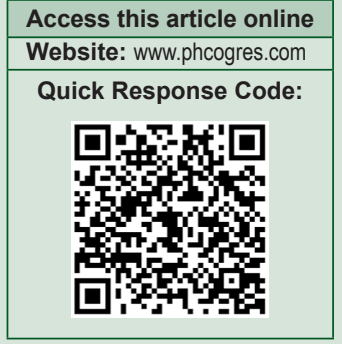

\section{INTRODUCTION}

Depression is a major contributor to global disability, ischemic heart disease, and incidence of suicide. ${ }^{[1]}$ It has been associated with emotional disorders and can cause disturbance of a set of cognitive functions, decrease quality of life, cause undesirable sociopsychological effects, and increase mortality rates. ${ }^{[2]}$ The global prevalence of depression and depressive symptoms has been increasing in recent decades, ${ }^{[3]}$ which represents a global prevalence of $7 \%$ for women and $4 \%$ for men. ${ }^{[4]}$ According to the World Health Organization (WHO), major depressive disorders accounted for the
This is an open access journal, and articles are distributed under the terms of the Creative Commons Attribution-NonCommercial-ShareAlike 4.0 License, which allows others to remix, tweak, and build upon the work non-commercially, as long as appropriate credit is given and the new creations are licensed under the identical terms.

For reprints contact: reprints@medknow.com

Cite this article as: Al-Harbi HR, Harthi S, Suliman M, Saadah O, Esmat A, Khan LM, et al. Antidepressant-like effects of barley (Hordeum vulgare) in a mouse model of reserpine-induced depression. Phcog Res 2020;12:199-204. 
second major illness in the world by the year 2020. ${ }^{[5]}$ By the year 2030, the WHO predicts that depression will be the third cause of disease burden worldwide. ${ }^{[6]}$ Patients with major depression have symptoms that reflect changes in brain monoamine neurotransmitters, specifically norepinephrine, serotonin, and dopamine. Despite significant developments, conventional treatment is effective only in one in three cases of mood disorder. Nowadays, the most common drugs prescribed are tricyclic antidepressants, selective serotonin reuptake inhibitors, monoamine oxidase inhibitors, noradrenergic reuptake inhibitors, specific serotonin-noradrenaline reuptake inhibitors, and recently introduced synaptic or GluN2B-selective extrasynaptic N-methyl-D-aspartate receptor inhibitors. ${ }^{[7,8]}$ Although many drugs appear to have an important role in cases of depression, many complaints that the drugs are not effective for all patients and have severe adverse effects if used for a long time. Therefore, it is desirable to seek fast-acting, better-tolerated, more effective, and fewer side effects of antidepressants. Therefore, there is a great need for alternative therapy for the treatment of depression. Several studies have demonstrated the use of complementary and alternative medicine among psychiatric disorders, especially depression. Therefore, barley was suggested in traditional Islamic medicine as a treatment for sadness. ${ }^{[9,10]}$

Barley (Hordeum vulgare) is one of the Poaceae (Gramineae) families. ${ }^{[1]}$ Barley grain contains phenolic compounds such as benzoic acid and cinnamic acid derivatives, proanthocyanidins, quinones, flavonols, chalcones, flavones, flavanones, and amino phenolic compounds, which act as an antioxidant agent. ${ }^{[12]}$ Moreover, it contains important minerals and vitamins such as $\mathrm{Ca}^{+}+, \mathrm{K}+, \mathrm{Mg}^{+}+$, and $\mathrm{P}^{[13]}$ and Vitamin $\mathrm{B} 12$, Vitamin C, folic acid, and tocopherol. ${ }^{[14]}$ The present study was performed to determine the effect of barely on behavioral pharmacotherapy.

\section{METHODS}

This study was conducted according to the guidelines of the Research Animal Care Committee-approved protocol at the King Fahd Medical Research Center. All studies conducted on animals were approved by the Bioethical Research Unit of Faculty of Medicine, King Abdulaziz University (Ref: 488-17).

\section{Subjects}

A total number of 60 male Albino Swiss mice (weighing 20-30 g), 6 weeks old, were housed in individual cages under environmentally controlled conditions (12-h light/12-h dark cycles and temperature $24^{\circ} \mathrm{C}$ ), with free access to rodent chow and water. Experiments occurred in the light portion of the cycle.

\section{Experimental design}

Barley seeds chosen were free from any fungal infection, then dried, and grounded. Fifteen grams of whole grain barley was boiled in $120 \mathrm{ml}$ of water for $5 \mathrm{~min}$ and then filtered. This dose administered was based on the nutritional dose suggested for an adult human. Barley (200 mg/kg, p. o.) were used in this experiment. Depression, learning, and memory were assessed with six behavioral paradigms: forced swimming test (FST), tail suspension test, hole-board test, novel place/object recognition, social interaction test, and spontaneous locomotor activity (SLMA) and stereotype movement. Animals received the required amount of barley and doses of the medications once daily for 4 weeks. Initial body weights and final body weights were measured at the beginning and end of the experiment, respectively. At the end of the experiment, animals of all groups were sacrificed under ether anesthetic and the brain was carefully removed from the skull, weighed, and then kept in cold normal saline. Seven study groups with 5 animals in each were tested at the end of a treatment period of 4 weeks. Groups were described in Figure 1.

\section{Behavioral tests \\ Forced swimming}

The test was carried out according to the method described by Porsolt et al. ${ }^{[15]}$ Briefly, $30 \mathrm{~min}$ after the last drug administration, mice were placed individually into the open glass cylinder (height: $45 \mathrm{~cm}$ and diameter: $20 \mathrm{~cm}$ ) filled with $20 \mathrm{~cm}$ of water maintained at $23-25^{\circ} \mathrm{C}$. Mice were left in the cylinder for $6 \mathrm{~min}$. Immobility was defined as mouse ceasing struggling, remaining floating motionless in water, except with only movement's necessary to keep its head above water. After $2 \mathrm{~s}$, the total of immobility duration in mice was recorded by EthoVision XT8 machine and scored by a blinded experimenter.

\section{Tail suspension test}

This method was carried out, as mentioned by Cryan et al. ${ }^{[16]}$ Mice were suspended by means of adhesive tape fixed approximately $1 \mathrm{~cm}$ from the caudal tip $5 \mathrm{~cm}$ above the floor. Immobility was defined as the absence of movement for $6 \mathrm{~min}$. The immobility was calculated (in seconds) for $6 \mathrm{~min}$. Mice considered immobile when they were completely motionless. The immobility duration in mice was recorded by EthoVision XT8 machine and scored by a blinded experimenter.

\section{Hole-board test}

The hole-board apparatus was used as described earlier by Sonavane et al. ${ }^{[17]}$ The hole-board is a chamber $(40 \mathrm{~cm} \times 40 \mathrm{~cm} \times 25 \mathrm{~cm})$ with 16 holes (each of $3 \mathrm{~cm}$ diameter) evenly distributed on the floor of the apparatus. The height of the apparatus was $25 \mathrm{~cm}$. The treated and control mice were left 60 min before the test and kept in the apparatus. The number of head pokes during 5 min was recorded.

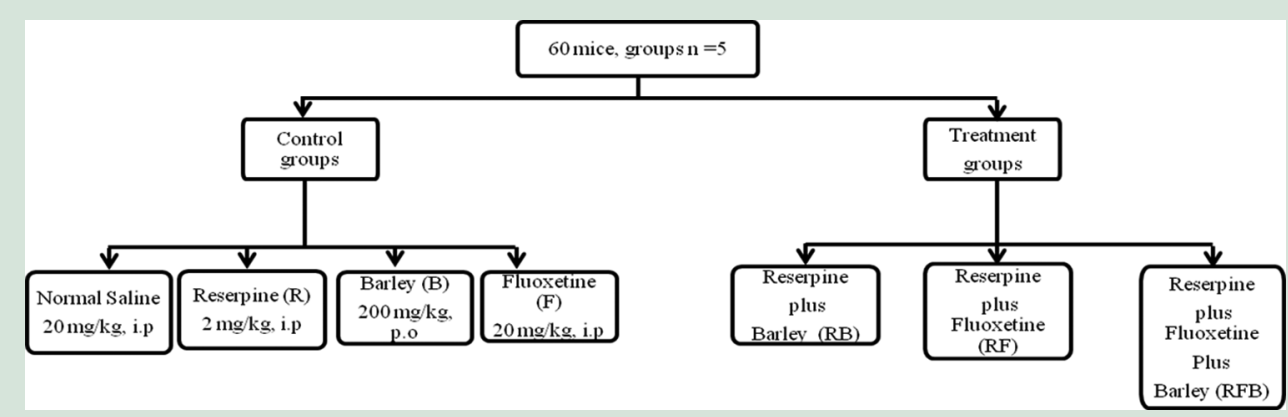

Figure 1: The experimental schematic, control groups: Normal saline (20 mg/kg, i. p.), Reserpine was dissolved in normal saline at a concentration of $2 \mathrm{mg} /$ $\mathrm{ml}$ and administered at a dose of $2 \mathrm{mg} / \mathrm{kg}$, i. p. Barley ( $200 \mathrm{mg} / \mathrm{kg}$, p. o) was administered by gavage and fluoxetine ( $20 \mathrm{mg} / \mathrm{kg}$, i. p.). Treatment gropus: Dual combination ( Reserpine and barley groups, Reserpine and fluoxetine), and the triple combination (Reserpine plus fluoxetine plus barley) 


\section{Novel place/object recognition}

This procedure evaluated the ability of mouse to recognize a novel object in the arena. This test consists of three stages: habituation stage, familiarization stage, and test stage. In the habituation stage, each animal allowed to explore the empty arena for $5 \mathrm{~min}$. After that, the animal returned to its holding cage. During familiarization stage, two identical objects were placed in an open-field arena and a single mouse allowed exploring them for $5 \mathrm{~min}$ then returned to its holding cage. During the test stage, the mouse returned to the open-field arena with two objects: one was the object used in familiarization test and the other was a novel object. Normal mouse should spend more time to the novel object. The time exploring the new object was measured with EthoVision video tracking software, version XT8 (Noldus Information Technology, Netherlands). ${ }^{[18]}$

\section{Social interaction test}

The test was conducted in open-field square arena $(65 \mathrm{~cm} \times 65 \mathrm{~cm}$ $\times 45 \mathrm{~cm}$ ) made of transparent plastic using video tracking system (EthoVision XT 8). Two identical conical-shaped wires enough to hold one mouse were used: one for familiar and the other for stranger mouse. The two conical-shaped wires were placed vertically on the opposite sides of the arena. The mouse to be tested allowed exploring the arena and empty shaped for $5 \mathrm{~min}$ and returned to its cage. One familiar mouse from the same cage of the tested mouse was put in the conical-shaped wire and the tested mouse was returned to the arena and permitted to explore and interact with the familiar mouse for five minutes, then returned to its cage. The stranger mouse was put in other conical-shaped wire, and the tested mouse returned to the arena and was permitted to explore and interact with stranger mouse for $5 \mathrm{~min}$. Normal mouse should interact with stranger mouse more than familiar one. ${ }^{[19]}$

\section{Spontaneous locomotor activity and stereotype movement}

The spontaneous locomotor activity of rats was monitored using automated Animex apparatus with four magnet-field coils (LKB Farad, Stockholm, Sweden) and housed in sound-attenuated chambers. The recording of spontaneous activity and stereotype movements started $15 \mathrm{~min}$ after the animals were placed individually into the Animex apparatus (habituation period). Data were collected in 5 sessions of 5 min each. ${ }^{[20]}$

\section{Drug and chemical agents}

Reserpine (methyl reserpate 3,4,5-trimethoxybenzoic acid ester) was obtained from (BDH Chemicals Ltd., England), dissolved in normal saline at a concentration of $2 \mathrm{mg} / \mathrm{ml}$, and administered at a dose of $2 \mathrm{mg} /$ $\mathrm{kg}$, i. p. This dose was selected based on pilot studies demonstrating depressive effects. Fluoxetine was from Sandoz Pharmaceuticals Inc (Sandoz Pharmaceuticals inc., Princeton US).

\section{Statistical analysis}

Statistical analysis was performed using the Statistical Package for the Social Sciences (SPSS, v23 software, IBM, Armonk, New York, USA). One-way analysis of variance was carried out. When equal variances were assumed, the least significant difference test was applied. Data were presented as mean \pm standard deviation (SD). $P<0.05$ was considered statistically significant.

\section{RESULTS}

\section{Effect of barley on body weight}

No significant differences in mean body weight were observed among the groups at the beginning of the experiment $(P=0.775)$. At the end of the experiment, there was a significant difference in the mean body weight in the reserpine ${ }^{\oplus}$, fluoxetine, and barley (RFB) group in comparison to all other groups $(P=0.01)$. Regarding brain weight, it was significantly higher in fluoxetine mice and RFB mice than in barley mice $(P=0.001$ and 0.012 , respectively), as demonstrated in Table 1 . The effects of barley on depression, learning, and memory in mice were also reported.

\section{Effects of barley on forced swimming test}

We measured immobilization time in theFST [Table2] after the completion of treatment. The mean duration of immobility was significantly higher in reserpine, fluoxetine, and group of reserpine and fluoxetine when compared with control mice (reserpine [R]: $236.53 \pm 38.66 \mathrm{~s}, P<0.05$ ). Moreover, immobilization time was significantly lower in barley mice alone or with reserpine (barley [B]: $44.60 \pm 12.21 \mathrm{~s}, \mathrm{RB}: 8.00 \pm 3.16 \mathrm{~s}$, $P<0.001)$. Furthermore, treatment with RFB significantly decreased immobilization time during the FST (RFB: $16.00 \pm 12.49$ s, $P<0.001$ ).

\section{Effect of barley on tail suspension test}

Mice treated with barley exhibited significant decreases in immobilization time during the TST when compared with the reserpine group (R: $116.6 \pm 57.43$, B: $26.21 .81 \mathrm{~s}, P<0.001)$. Moreover, treatment with $\mathrm{RB}$ and RFB significantly decreased immobilization time during the FST (RB: $62.10 \pm 53.62 \mathrm{~s}, \mathrm{RFB}: 93.50 \pm 04 \mathrm{~s}, P<0.001$ ), as shown in Table 2.

Table 1: The initial final body and brain weights in different treated and untreated groups of mice

\begin{tabular}{|c|c|c|c|c|c|c|c|c|}
\hline & C & $\mathbf{R}$ & B & $\mathbf{F}$ & RB & RF & RFB & $P$ \\
\hline Initial brain weight & $28.80 \pm 1.8$ & $29.55 \pm 0.72$ & $27.85 \pm 2.5$ & $27.94 \pm 1.99$ & $28.70 \pm 0.81$ & $30.12 \pm 0.72$ & $29.10 \pm 1.89$ & 0.775 \\
\hline Final brain weight & $29.43 \pm 2.40$ & $27.64 \pm 2.62$ & $27.98 \pm 3.47$ & $28.40 \pm 1.01$ & $29.46 \pm 1.97$ & $29.08 \pm 0.87$ & $23.39 \pm 1.08^{* * *}$ & 0.001 \\
\hline Brain weight & $0.46 \pm 0.06$ & $0.46 \pm 0.02$ & $0.41 \pm 0.06$ & $0.52 \pm 0.03^{\star}$ & $0.44 \pm 0.05$ & $0.42 \pm 0.04$ & $0.45 \pm 0.01^{*}$ & 0.061 \\
\hline
\end{tabular}

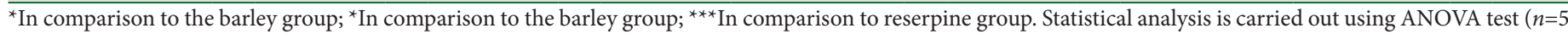

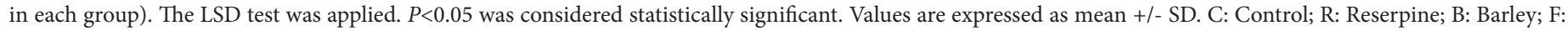

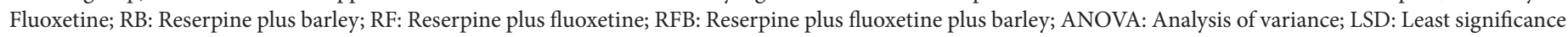
difference; SD: Standard deviation

Table 2: The duration of immobility (s) during forced swimming test and tail suspension test in addition to the number of head poking

\begin{tabular}{|c|c|c|c|c|c|c|c|c|}
\hline & $C$ & $\mathbf{R}$ & B & $F$ & RB & RF & RFB & $P$ \\
\hline FST (s) & $225.62 \pm 15.24$ & $236.53 \pm 38.66$ & $225.84 \pm 29.28^{*}$ & $230.99 \pm 36.58$ & $215.46 \pm 52.56^{*}$ & $238.59 \pm 15.81$ & $230.14 \pm 12.92^{*}$ & 0.05 \\
\hline Number of head poking & $23.00 \pm 3.53$ & $25.00 \pm 16.71$ & $44.60 \pm 12.21$ & $20.40 \pm 8.11$ & $34.60 \pm 18.78$ & $29.31 \pm 13.14$ & $30.00 \pm 12.49$ & 0.001 \\
\hline TST (s) & $96 \pm 64.5$ & $116.6 \pm 57.43$ & $26 \pm 21.81$ & $75.4 \pm 71.4$ & $62.12 \pm 53.62$ & $78.2 \pm 37.7$ & $93.52 \pm 66.04$ & 0.001 \\
\hline
\end{tabular}

${ }^{\star}$ In comparison to the barley group. Statistical analysis is carried out using ANOVA-test ( $n=5$ in each group). The LSD test was applied. $P<0.05$ was considered

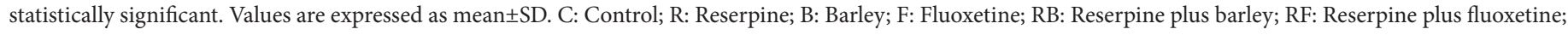

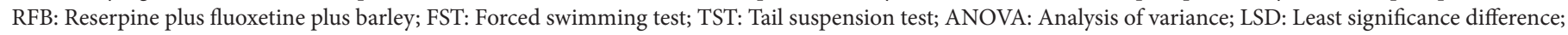
SD: Standard deviation 


\section{Effect of barley on head poking test}

Table 2 also shows that mice treated with barley significantly decreased the number of head poking compared with the reserpine group (R: $25.00 \pm 16.71,44.60 \pm 12.21, P<0.001$ ). In addition, treatment with RB and RFB significantly decreased a number of head poking (RB: $34.60 \pm 18.78$, RFB: $30.00 \pm 12.49, P<0.001$ ).

\section{Effect of barley on social interaction test and novel object recognition test}

The principle of these tests is to evaluate two critical aspects of social behavior, such as social affiliation, as well as social memory and novelty. The mean interaction frequency and interaction time for the social interaction test are shown in Table 3. Mice treated with reserpine revealed a significant increase in the distance covered toward familiar ones compared with those of the control and barley groups ( $\mathrm{R}$ : $169.14 \pm 131.89 \mathrm{~cm}, \mathrm{C}: 1327.20 \pm 518.09 \mathrm{~cm}, \mathrm{~B}: 1320.80 \pm 528.96 \mathrm{~cm}$, $P<0.001)$. Moreover, those treated with RB and RFB showed a significant decrease in the distance covered toward familiar ones compared with those of the reserpine group (RB: $452.57 \pm 425.55 \mathrm{~cm}$, RFB: $518.93 \pm 344.44 \mathrm{~cm}, P<0.001)$. Regarding the distance measured to the stranger mice, there was a significant decrease in the distance covered in those treated with barley, barley and fluoxetine, and reserpine, fluoxetine, and barley (RFB) compared with the reserpine group (R:295.66 \pm 149.18 $\mathrm{cm}, \mathrm{B}: 1768.7 \pm 1005.57 \mathrm{~cm}, \mathrm{BF}: 645.35 \pm 408.42$, RFB: $341.80 \pm 357.61 \mathrm{~cm}$, $P<0.001)$. In addition, mice treated with barley alone spend significantly more time in the compartment with familiar and stranger one compared to the reserpine group alone (familiar mouse $-\mathrm{R}$ : $169.14 \pm 131.89$ s, B: $1320.8 \pm 528.96$ s; stranger mouse - R: $295.66 \pm 149.18$ s, B: $1768.7 \pm 1005.57 \mathrm{~s}, P<0.001$, respectively). Mice treated with barley and reserpine (familiar mouse - BR: $452.57 \pm 425.55 \mathrm{~s}$, stranger mouse - BR: $645.35 \pm 408.42 \mathrm{~s}, P<0.001$, respectively) spend significantly more time in the compartment with familiar and stranger one compared to the reserpine group alone.

The mean exploration time in the novel place recognition test is shown in Table 4. The time spent to explore the novel object by mice of groups of barely and barley and fluoxetine did not show any significant difference as compared to time spent by mice treated with barley alone. On the other hand, there was a significant decrease in the time spent by mice in groups of reserpine, reserpine and imipramine, reserpine and barley and reserpine, and imipramine and barley as compared to those of the barley group $(P<0.001)$

\section{Effect of barley on spontaneous locomotor movement and stereotype movements}

Tables 5 and 6 demonstrate that the spontaneous locomotor movements (SLMA) and the stereotype movements. The average time spent to explore SLMA and stereotype movements were significantly decreased in groups of reserpine (R: $1733.92 \pm 88.71$ ), in comparison to those of the barley groups (B: $1452.68 \pm 522.91 \mathrm{~cm}$; RB: $69.72 \pm 83.268 \mathrm{~cm}$; RFB: $64.88 \pm 48.994 \mathrm{~cm}, P<0.001)$. Likewise, total distance traveled was also rescued by fluoxetine.

\section{DISCUSSION}

In the present study, we investigated the antidepressant effects of barley in mice with reserpine-induced depression. Our results revealed that barley can ameliorate depressive-like behaviors. There are several causes of depression; however, the pathophysiology of the disorder remains to be fully elucidated. Many hypotheses have been proposed regarding the basis of depression, such as hypothalamic-pituitary-adrenal axis hyperactivity; disturbances in gamma-aminobutyric acid transmission, monoamine, and glutamate; neurotrophic factor dysfunction; and glial pathology. ${ }^{[21-23]}$ Monoamines are transported into presynaptic vesicles

Table 3: The mean distance $(\mathrm{cm})$ covered by mice in different groups using social interaction test

\begin{tabular}{ccccccccc}
\hline & C & R & B & F & RB & RF & RFB & $P$ \\
\hline Familiar mice & $1327.2 \pm 518.09$ & $169.14 \pm 131.89^{* *}$ & $1320.8 \pm 528.96$ & $1526.8 \pm 311.65^{* * *}$ & $452.57 \pm 425.55$ & $164.57 \pm 86.24^{* * *}$ & $518.93 \pm 344.44^{* * *}$ & 0.001 \\
Stranger mice & $1137.8 \pm 618.53$ & $295.66 \pm 149.18^{* *}$ & $1768.7 \pm 1005.57$ & $276.34 \pm 113.05^{* *}$ & $645.35 \pm 408.42^{* *}$ & $532.51 \pm 145.36^{* *}$ & $341.80 \pm 357.61^{* *}$ & 0.001 \\
\hline
\end{tabular}

${ }^{* *}$ In comparison to barley group; ${ }^{* * *}$ In comparison to reserpine group. Statistical analysis is carried out using ANOVA-test ( $n=5$ in each group). The LSD test was applied. $P<0.05$ was considered significant. Values are expressed as mean \pm SD. C: Control; R: Reserpine; B: Barley; F: Fluoxetine; RB: Reserpine plus barley; RF: Reserpine plus fluoxetine; RFB: Reserpine plus fluoxetine plus barley; ANOVA: Analysis of variance; LSD: Least significance difference; SD: Standard deviation

Table 4: The mean time spent (s) by mice in different groups for recognition of familiar or novel objects using novel object recognition test

\begin{tabular}{|c|c|c|c|c|c|c|c|c|}
\hline & $C$ & $R$ & B & $F$ & RB & RF & RFB & $P$ \\
\hline & $2 \pm$ & $14 \pm$ & $0.8 \pm$ & $8 \pm 3$ & +4 & $57+8$ & 3.9 & 0.001 \\
\hline
\end{tabular}

**In comparison to barley; ${ }^{* * *}$ In comparison to reserpine group. Statistical analysis is carried out using ANOVA-test $(n=5$ in each group). The LSD test was applied. $P<0.05$ was considered significant. Values are expressed as mean \pm SD. C: Control; R: Reserpine; B: Barley; F: Fluoxetine; RB: Reserpine plus barley; RF: Reserpine plus fluoxetine; RFB: Reserpine plus fluoxetine plus barley; ANOVA: Analysis of variance; LSD: Least significance difference; SD: Standard deviation

Table 5: Spontaneous locomotor movement $(\mathrm{cm})$ during 5 sessions in different groups of mice

\begin{tabular}{lcccccc} 
Groups & Session 1 & Session 2 & Session 3 & Session 4 & Session 5 & Average of all sessions \\
\hline C & $1101.40 \pm 360.42$ & $811.00 \pm 532.85$ & $872.2 \pm 822.79$ & $653.60 \pm 513.88$ & $620.80 \pm 432.44$ & $1432.6 \pm 532.476$ \\
R & $246.40 \pm 210.09$ & $8240.00 \pm 107.28$ & $86.80 \pm 82.91$ & $6.40 \pm 9.73$ & $15.00 \pm 33.54$ & $1733.92 \pm 88.71$ \\
B & $1194.4 \pm 327.01$ & $1273.0 \pm 551.34$ & $826.20 \pm 815.11$ & $933.80 \pm 695.37$ & $506.00 \pm 225.72$ & $1452.68 \pm 522.91^{* * *}$ \\
F & $1118.6 \pm 242.39$ & $1019.4 \pm 179.42$ & $718.20 \pm 427.99$ & $761.80 \pm \pm 43.19$ & $427.40 \pm 197.22$ & $1236.48 \pm 298.042^{* * *}$ \\
RB & $117.80 \pm 79.52$ & $109.40 \pm 150.42$ & $56.40 \pm 126.11$ & $19.40 \pm 43.37$ & $7.60 \pm 16.92$ & $69.72 \pm 83.268^{* * *}$ \\
RF & $225.20 \pm 180.40$ & $129.40 \pm 179.42$ & $87.00 \pm 85.77$ & $11.00 \pm 10.77$ & 0 & $90.52 \pm 91.272^{* * *}$ \\
RFB & $141.20 \pm 79.87$ & $136.80 \pm 81.89$ & $41.40 \pm 77.78$ & $5.00 \pm 5.43$ & 0 & $64.88 \pm 48.994^{* * *}$ \\
\hline
\end{tabular}

${ }_{* * *}$ In comparison to reserpine. Statistical analysis is carried out using ANOVA-test ( $n=5$ in each group). The LSD test was applied. $P<0.05$ was considered significant. Values are expressed as mean \pm SD. C: Control; R: Reserpine; B: Barley; F: Fluoxetine; RB: Reserpine plus barley; RF: Reserpine plus fluoxetine; RFB: Reserpine plus fluoxetine plus barley; ANOVA: Analysis of variance; LSD: Least significance difference; SD: Standard deviation 
HAMAD AL-HARBI, et al:: Antidepressant Effect of Barley Decoction in Mice

Table 6: Stereotype movements in 5 sessions

\begin{tabular}{lcccccc} 
Groups & Session 1 & Session 2 & Session 3 & Session 4 & Session 5 & Average of all sessions \\
\hline C & $32.00 \pm 35.90$ & $35.20 \pm 34.24$ & $33.60 \pm 31.30$ & $25.60 \pm 26.12$ & $33.40 \pm 31.95$ & $65.36 \pm 31.90$ \\
R & $35.20 \pm 22.70$ & $23.00 \pm 22.16$ & $28.80 \pm 15.49$ & $15.20 \pm 14.27$ & $12.00 \pm 11.31$ & $34.84 \pm 17.19$ \\
B & $34.60 \pm 30.38$ & $32.80 \pm 38.79$ & $25.40 \pm 24.82$ & $25.60 \pm 23.37$ & $26.60 \pm 26.48$ & $55.6 \pm 28.77 * * *$ \\
F & $33.00 \pm 29.55$ & $31.60 \pm 36.05$ & $23.60 \pm 24.61$ & $24.80 \pm 21.75$ & $25.00 \pm 24.77$ & $52.6 \pm 27.35$ \\
RB & $28.60 \pm 10.83$ & $26.20 \pm 17.75$ & $18.00 \pm 17.24$ & $17.40 \pm 14.75$ & $14.20 \pm 14.73$ & $35.08 \pm 15.06$ \\
RF & $1129.8 \pm 322.02$ & $973.40 \pm 15.19$ & $806.40 \pm 429.18$ & $738.80 \pm 413.29$ & $465.20 \pm 229.43$ & $1287.92 \pm 281.82^{* * *}$ \\
RFB & $40.40 \pm 24.75$ & $33.20 \pm 22.78$ & $19.40 \pm 15.32$ & $16.40 \pm 13.95$ & $15.60 \pm 16.456$ & $40.6 \pm 18.65$ \\
\hline
\end{tabular}

${ }_{* * *}$ In comparison to reserpine. Values are expressed as mean \pm SD. Statistical analysis was carried out using ANOVA-test ( $n=5$ in each group). The LSD test was applied. $P<0.05$ was considered significant. C: Control; R: Reserpine; B: Barley; F: Fluoxetine; RB: Reserpine plus barley; RF: Reserpine plus fluoxetine; RFB: Reserpine plus fluoxetine plus barley; ANOVA: Analysis of variance; LSD: Least significance difference; SD: Standard deviation

through the vesicular monoamine transporter, which is blocked by reserpine. Mice with reserpine-induced depression due to monoamine depletion exhibit anxiety and depressive-like behaviors, such as increased immobility time and decreased locomotor activity in behavioral tests, relative to results observed in control mice. Moreover, previous studies have demonstrated that mice treated with reserpine show increased levels of plasma corticosterone and pro-inflammatory cytokines in the brain. ${ }^{[24,25]}$ In this study, reserpine was used to induce the reactive type of depression in mice. Similarly, El-Sisi (2011) mentioned that intraperitoneal injection of reserpine $(10 \mathrm{mg} / \mathrm{kg} / \mathrm{b}$. wt) for 2 weeks induced a significant decrease in catecholamine levels in both cortex and hippocampus. ${ }^{[26]}$ Moreover, reserpine, as a sympatholytic drug depletes catecholamine in peripheral nervous tissues and in the brain, ${ }^{[27]}$ can irreversibly inhibit the vesicular uptake of monoamines, including noradrenaline, dopamine, and 5-hydroxytryptamine, which depletes monoamines in the brain and produces depression-like syndrome in animals. ${ }^{[28]}$ In the present study, we have tried to demonstrate that barley is able to reverse the depression in mice induced by reserpine.

Barley (H. vulgare) was used in this study as a natural medicine. There have been a number of unpublished but reputable concerns on the antidepressant effects of the young green barley leaf in Japan. ${ }^{[29]}$ Our results showed that no significant difference was revealed between the initial body weights of all experimental groups. However, groups of RFB and reserpine $e^{\infty}$ showed a significant reduction in the final body weight with comparison to other groups. Furthermore, inconsistent results still exist in the literature with reference to the effect of fluoxetine on body weight changes. Some studies reported various weight reductions with fluoxetine. ${ }^{[30]}$ Moreover, barley successfully ameliorated depressive-like behaviors in reserpine-treated mice, as indicated by significant reductions in immobility time as expressed in mean \pm SD in FST and TST. It was reported that forced swim test and tail suspension test are the most validated tests. They are an aversive stressful situation that generates behavioral despair as immobility. ${ }^{[16]}$ The FST is an animal model that is used as an experimental paradigm for the assessment of despair/depression-like behavior. It is also commonly used as a screening test for the antidepressant properties of drugs. Our results reported that barley significantly reduces immobility duration in the mouse FST. The mean duration of immobility was significantly higher in reserpine, fluoxetine, and group of reserpine and fluoxetine when compared with control mice. Moreover, immobilization time was significantly lower in barley mice alone or with reserpine. Furthermore, treatment with RFB significantly decreased immobilization time during the FST. Mice treated with barley exhibited significant decreases in immobilization time during the TST when compared with the reserpine group. Moreover, treatment with reserpine plus barley and fluoxetine plus barley followed by reserpine significantly decreased immobilization time during the FST, indicating that the barley was effective for producing an antidepressant-like effect in these behavioral models. Furthermore, mice treated with barley significantly decreased the number of head poking compared with the reserpine group. In addition, treatment with barley followed by reserpine and fluoxetine and barley followed by reserpine significantly decreased a number of head poking, indicating that the head-dipping behavior was sensitive to changes in the emotional state of the animal and suggesting that the expression of an anxiolytic state may be reflected by an increase in head-dipping behavior. ${ }^{[31]}$ Mice treated with reserpine revealed a significant increase in the distance covered toward familiar ones compared with those of the control and barley groups. Moreover, those treated with barley followed by reserpine and fluoxetine plus barley followed by reserpine demonstrated a significant decrease in the distance covered toward familiar ones compared with those of the reserpine group. Regarding the distance measured to the stranger mice, there was a significant decrease in the distance covered in those treated with barley, barley and fluoxetine, and RFB compared with the reserpine group. In addition, mice treated with barley alone spend significantly more time in the compartment with familiar and stranger one compared to the reserpine group alone. Mice treated with barley and reserpine spend significantly more time in the compartment with familiar and stranger one as compared to the reserpine group alone.

The time spent to explore the novel object by mice of the barley group and barley followed by reserpine did not show any significant difference as compared to time spent by mice treated with barley alone. On the other hand, Spontaneous locomotor movements (SLMA) and the stereotype movements demonstrated that, the time spent to explore SLMA and stereotype movements were significantly decreased in groups of reserpine, in comparison to those of the barley groups. Several studies have suggested that alpha (2)-adrenoceptors strongly affect monoaminergic neurotransmission by enhancing not only noradrenergic but also serotonergic firing rates. ${ }^{[10,32]}$ Andrade and Rao (35) hypothesized that antidepressant drugs are associated with the induction of neuroplasticity in structures such as the hippocampus and prefrontal cortex: There is stimulation of neurogenesis, gliogenesis, dendritic arborization, and new synapse formation. ${ }^{[33]}$ These changes may underlie the mechanisms of antidepressant response because their time course of development parallels the time course of antidepressant action, since they reverse the neurohistological effects of stress and they may allow the relearning of healthier cognitions, emotional responses, and behavioral expressions.

\section{CONCLUSION}

Taken together, our results reported that oral administration of barley is able to produce an antidepressant-like effect in various behavioral tests. Furthermore, future efforts will be focused on further elucidation of the mechanisms of action of barley at the molecular level underlying complex behaviors.

\section{Acknowledgements}

The authors thankfully acknowledge Mrs. Hana Khalaf and her team at King Fahd Medical Research Center, King Abdulaziz University, Jeddah, for providing the technical assistance. 


\section{Financial support and sponsorship}

Nil.

\section{Conflicts of interest}

There are no conflicts of interest.

\section{REFERENCES}

1. World Health Organization. Other Common Mental Disorders: Global Health Estimates. Geneva: World Health Organization; 2017. p. 1-24.

2. Blazer DG. Depression and social support in late life: A clear but not obvious relationship, Aging \& Mental Health 2005;9:497-499. DOI: 10.1080/13607860500294266.

3. Kuo DC, Tran M, Shah AA, Matorin A. Depression and the suicidal patient. Emerg Med Clin North Am 2015;33:765-78.

4. Steel Z, Marnane C, Iranpour C, Chey T, Jackson JW, Patel V, et al. The global prevalence of common mental disorders: A systematic review and meta-analysis 1980-2013. Int J Epidemiol 2014;43:476-93.

5. Murray CJ, Lopez AD. Alternative projections of mortality and disability by cause 1990-2020: Global Burden of Disease Study. Lancet 1997;349:1498-504.

6. Malhi GS, Mann JJ. Depression. Lancet 2018;392:2299-312.

7. David N, Simon D, Wilson Sue BA. Psychotropic drugs. In Bennett MJ, Brown MJ, Sharma P (editors), Cliinical Pharmacology. 11th ed. London: Churchill Livingstone; 2012. p. 311-22. https://doi.org/10.1016/B978-0-70204084-9.00059-8.

8. Zanos P, Gould TD. Mechanisms of ketamine action as an antidepressant. Mol Psychiatry 2018;23:801-11.

9. Al-Bukhari MI. Food. Hadith 5417. In: Al-Bukhari S. 1st ed. Arabic, Damascus: Dar Ibn Kather; 2002. p. 1379.

10. Khalifa NA. The effect of barley broth (Talbinah) in management of depression. Int J Food Nutr Public Health 2017;9:111.

11. Hashemi JM. Biomedical effects of barley - A review. NY Sci J 2015;8:52-5.

12. Omwamba M, Hu Q. Antioxidant activity in barley (Hordeum vulgare L.) grains roasted in a microwave oven under conditions optimized using response surface methodology. J Food Sci 2010;75:C66-73.

13. Stewart A, Nield H, Lott JN. An investigation of the mineral content of barley grains and seedlings. Plant Physiol 1988;86:93-7

14. Granda L, Rosero A, Benešová K, Pluháčková H, Neuwirthová J, Cerkal R Content of selected vitamins and antioxidants in colored and nonpigmented varieties of Quinoa, Barley, and Wheat Grains. J Food Sci 2018;83:2439-47.

15. Porsolt RD, Bertin A, Jalfre M. Behavioral despair in mice: A primary screening test for antidepressants. Arch Int Pharmacodyn Ther 1977;229:327-36.

16. Cryan JF, Valentino RJ, Lucki I. Assessing substrates underlying the behavioral effects of antidepressants using the modified rat forced swimming test. Neurosci Biobehav Rev 2005;29:547-69.

17. Sonavane GS, Sarveiya VP, Kasture VS, Kasture SB. Anxiogenic activity of Myristica fragrans seeds. Pharmacol Biochem Behav 2002;71:239-44.

18. Antunes M, Biala G. The novel object recognition memory: Neurobiology, test procedure, and its modifications. Cogn Process 2012;13:93-110.

19. Page DT, Kuti OJ, Sur M. Computerized assessment of social approach behavior in mouse. Front Behav Neurosci 2009;3:48.

20. Savi MM, Obradovi DI, Ugrešić ND, Bokonji DR. The influence of diazepam on atropine reversal of behavioural impairment in dichlorvos-treated rats. Pharmacol Toxicol 2003;93:211-8.

21. Stetler C, Miller GE. Depression and hypothalamic-pituitary-adrenal activation: A quantitative summary of four decades of research. Psychosom Med $2011 ; 73: 114-26$

22. Schiepers OJ, Wichers MC, Maes M. Cytokines and major depression. Prog Neuropsychopharmacol Biol Psychiatry 2005;29:201-17.

23. Hasler G. Pathophysiology of depression: Do we have any solid evidence of interest to clinicians? World Psychiatry 2010:9:155-61.

24. Szelényi J, Vizi ES. The catecholamine cytokine balance: Interaction between the brain and the immune system. Ann NY Acad Sci 2007;1113:311-24.

25. Revzin AM, Maickel RP, Costa E. Effects of reserpine and benzoquinolizines on limbic system excitability, brain amine storage and plasma corticosterone level in rats. Life Sci (1962) 1962;1:699-707.

26. El-Sisi SF. Evaluation of the antidepressant like effect for some natural supplements against reserpine induced behavioral depression in mice. NY Sci J 2011;4:93-104.

27. Bao S, Fei J, Shen J, Gong SJ, Fang H, Husband AJ. Reserpine-induced mode of stress suppresses mucosal immunity. Immunol Cell Biol 2006;84:537-42.

28. Yu L, Jiang X, Liao M, Ma R, Yu T. Antidepressant-like effect of tetramethylpyrazine in mice and rats. Neurosci Med. 2011:2:142-8.

29. Zeng Y, Pu X, Yang J, Du J, Yang X, Li X, et al. Preventive and Therapeutic Role of Functional Ingredients of Barley Grass for Chronic Diseases in Human Beings. Oxid Med Cell Longev 2018;2018:3232080.

30. Afkhami-Ardekani M, Sedghi H. Effect of fluoxetine on weight reduction in obese patients. Indian J Clin Biochem 2005;20:135-8.

31. Takeda $H$, Tsuji M, Matsumiya T. Changes in head-dipping behavior in the hole-board test reflect the anxiogenic and/or anxiolytic state in mice. Eur $\mathrm{J}$ Pharmacol 1998;350:21-9.

32. Dhir A, Kulkarni SK. Effect of addition of yohimbine (alpha-2-receptor antagonist) to the antidepressant activity of fluoxetine or venlafaxine in the mouse forced swim test. Pharmacology 2007;80:239-43.

33. Andrade C, Rao NS. How antidepressant drugs act: A primer on neuroplasticity as the eventual mediator of antidepressant efficacy. Indian J Psychiatry 2010;52:378-86. 